\title{
Anesthesia and Respiratory Disorders
}

\author{
Pranav Bansal $^{1}$, Mayuri Gupta ${ }^{2}$, Ishrat Yousuf ${ }^{2}$ \\ ${ }^{1}$ Professor \& Head, ${ }^{2}$ Resident: Department of Anesthesiology \& Critical Care, BPS Govt Medical College, \\ Khanpur Kalan, Sonipat, Haryana)
}

A 56- year old male patient, chronic smoker for the past 30 years, is known case of chronic obstructive pulmonary disease. pre-operative stabilization with inhaled bronchodilators, oral antibiotics and mucolytics the patient is posted for laparoscopic cholecystectomy. After induction of general anesthesia and securing airway with endotracheal tube, the surgery is started. After insufflation of abdomen with carbon-dioxide, the oxygen saturation falls and ventilation becomes difficult as judged by difficulty in pressing reservoir bag in Bain's circuit. $Q$ 1.Which of the following is not observed during perioperative bronchospasm:-

(A) Plateau pressure may remain unchanged

(B) Decreased exhaled tidal volumes

(C) Ultrasonography may aid in detecting bronchospasm

(D) Slow rising waveform on the capnograph

Q 2. Which of the following capnogram images correlates with airway obstruction/ bronchospasm?

(A)

(C)

(D)

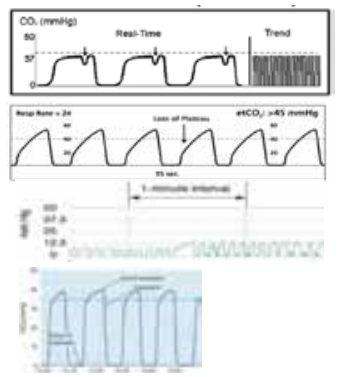

Q 3. Which of the following is not observed during intraoperative pulmonary embolism?

(A) Wide fixed splitting of the S2 heart sound

(B) A rise in end-tidal $\mathrm{CO}_{2}$ concentration

(C) Rapidly developing hypoxemia, and or bronchospasm

(D) Unexplained tachycardia and hypotension

Q 4. The presence of following is suggestive of chronic obstructive lung disease?

(A) Normal maximum mid expiratory flow (MMEF)
(B) Decline in closing capacity

(C) Increased residual volume

(D) Decreased total lung capacity

Q 5. Which of the following is incorrect regarding restrictive lung disease?

(A) Decrease in lung compliance and lung volumes

(B) Preservation of normal expiratory flow rates

(C) $\mathrm{FEV}_{1} / \mathrm{FVC}$ ratio is normal

(D) Forced expiratory volume in the first second of exhalation $\left(\mathrm{FEV}_{1}\right)$ is reduced and forced vital capacity (FVC) is increased

Q 6. Following bronchodilators therapy in an asthmatic patient, the reversibility of $\mathrm{FEV}_{1}$ is expected to be approximately:-
(A) $12-15 \%$
(B) $20-30 \%$
(C) $30-40 \%$
(D) $40-50 \%$

Q 7. Which of the following is not a feature of cystic fibrosis?

(A) Presence of pneumonia, wheezing and bronchiectasis

(B) PFT reveals reveal decreased vital capacity and expiratory flow rate

(C) Malabsorption syndrome

(D) Decrease in residual volume

Q 8. Which of the following flow volume loops is observed in a patient with tracheal stenosis?
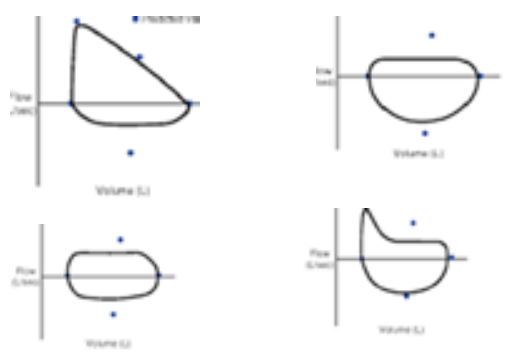

Q 9. Which of the following is true regarding emphysema?

(A) Centrilobular (or centriacinar) form is associated with $\alpha 1$-antitrypsin deficiency, and 
has predominantly a lower lobe distribution.

(B) Panlobular (or panacinar) form is more closely associated with tobacco smoking, and has predominantly an upper lobe distribution.

(C) Patients have increase in RV, FRC, TLC, and the RV/TLC ratio

(D) Diffusion capacity of lung is normal and well preserved till late stages

Q 10. Which of the following is true regarding mechanical ventilation in a patient with COPD?

(A) Air trapping leads to hypertension due to overinflated alveoli

(B) Increased ratio of inspiratory to expiratory time improves oxygenation

(C) Extrinsic PEEP is not applied to avoid overinflation

(D) Supplementation of higher $\mathrm{FiO}_{2}$ can improve the hypoxic ventilatory drive

\section{ANSWERS}

A 1. (C): Ultrasonography has no role in diagnosis of bronchospasm. Peak airway pressures show a rise during bronchospasm or upper airway disorders leading to a rise in dynamic compliance. The static compliance of alveoli are not altered due to airway diseases, hence plateau pressures remain normal.

A 2. (B): Capnograph develops "shark fin appearance" due to slow rise in waveform during airway obstruction and bronchospasm. Image $\mathrm{C}$ denotes return of spontaneous circulation while image $\mathrm{D}$ reveals hypercapnia where $\mathrm{CO}_{2}$ levels are high despite hyperventilation.

A 3. (B): A fall in end tidal $\mathrm{CO}_{2}$ readings is observed $\mathrm{n}$ pulmonary embolism due to obstruction of pulmonary veins leading due impaired perfusion and ventilation in alveoli.

A 4. (C): Obstructive lung disease shows following findings during lung function test:-

- Normal or increased total lung capacity

- Normal or increased vital capacity

- Decreased maximum mid expiratory flow rate

- Increased closing capacity

A 5. (D): FEV1 and FVC both are reduced in restrictive lung disease while FEV1 is reduced with a normal FVC in obstructive lung disease.
A 6. (A) Reversibility following therapeutic intervention is a characteristic feature of bronchial asthma not COPD. Following treatment with bronchodilators there is improvement in $\mathrm{FEV}_{1}$ by 12 $15 \%$ or $200 \mathrm{ml}$ noted on repeating spirometry after 15-30 minutes.

A 7. (D): Cystic fibrosis is associated with an increase in residual volume and airway resistance

A 8. (C): Patients with tracheal stenosis have fixed airway obstruction and show a blunted loop during both inspiration and expiration.

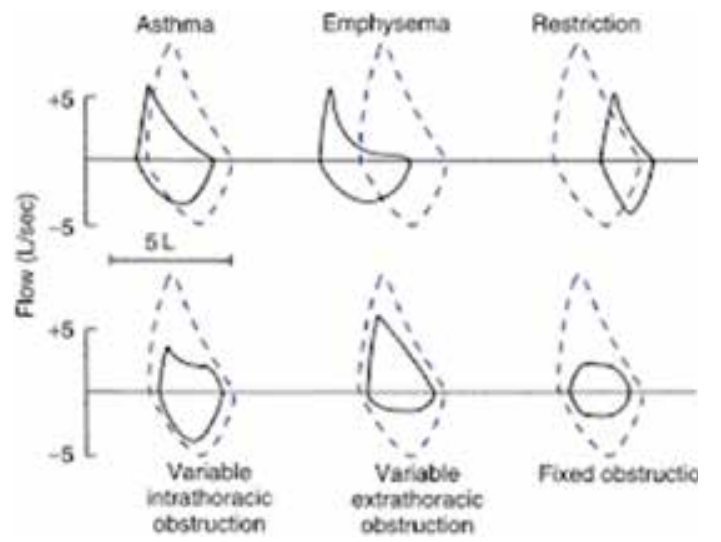

A 9. (C): Centrilobular (or centriacinar) form results from dilation or destruction of the respiratory bronchioles, is more closely associated with tobacco smoking, and has predominantly an upper lobe distribution. The panlobular (or panacinar) form results in a more even dilation and destruction of the entire acinus, is associated with $\alpha 1$-antitrypsin deficiency, and has predominantly a lower lobe distribution.

A 10. (C) During mechanical ventilation in a patient with COPD:-

- Air trapping leads to hypotension due to decreased venous return

- Inspiratory to expiratory time ratio is usually decreased to limit dynamic hyperinflation and maintain $\mathrm{PCO}_{2}$ levels

- Extrinsic PEEP is not applied to avoid overinflation due to presence of auto/ intrinsic PEEP

- Supplementation of higher $\mathrm{FiO}_{2}$ can lead to loss of hypoxic ventilatory drive in patients with COPD 